\title{
Synthesis and Biological Activity of (2-Substituted-4-methylthiazol-5-yl) (4-substituted piperazin-1-yl)methanone Derivatives
}

\author{
Pravin C. Mhaske*, Shivaji H. Shelke ${ }^{\dagger}$, Hemant N. Raundal ${ }^{\dagger}$, and Rahul P. Jadhav ${ }^{\dagger}$ \\ Post Graduate Department of Chemistry, S. P. Mandali's Sir Parashurambahu College, \\ Tilak Road, Pune, India 411 030, (Affiliated to University of Pune). \\ ${ }^{*}$ E-mail: mhaskepc18@gmail.com \\ ${ }^{\dagger}$ Department of Chemistry, HPT Arts and RYK Science College, Nashik India-422 005
}

(Received September 2, 2013; Accepted December 17, 2013)

\begin{abstract}
In the present study a novel series of (2-substituted-4-methylthiazol-5-yl)(4-substituted piperazin-1-yl)methanone derivatives were synthesized by reaction of 2-substituted-4-methylthiazole-5-carboxylic acid with N-substituted benzyl piperazine by using 1-ethyl-3-[3-(dimethylamino)propyl]carbodiimide hydrochloride (EDCl) and 1-hydroxybenzotriazole (HOBt) in DMF. All the newly synthesized compounds were characterized by spectral methods. The title compounds were screened for in vitro antibacterial activity. Most of the compounds show moderate to good antimicrobial activity.
\end{abstract}

Key words: Thiazole, Piperazine, EDCl-HOBt, Antimicrobial activity

Thiazole and their derivatives have attracted continuing interest over the years because of their varied biological activities. ${ }^{1}$ 1,3-Thiazole nucleus containing compounds have exhibited a broad range of biological activities. ${ }^{2-13} 5$ Azole carboxamide derivatives showed antianoxic activity. ${ }^{14}$ The aryl imidazolyl carboxamide derivatives were shown to be cannabinoid $\mathrm{CB}_{1}$ receptor antagonist. ${ }^{15,16}$ Based on these observations, a series of (2-substituted-4-methylthiazol-5-yl)(4-substituted piperazin-1-yl)methanone derivatives were synthesized and subjected to microbial screening.

The synthesis of (2-methyl, phenyl or benzyl-substituted4-methylthiazol-5-yl)(4-phenyl or benzyl substituted piperazin-1-yl)methanone is illustrated in Scheme 1. Ethyl-2chloro-3-oxobutanoate 1 on reaction with methyl, phenyl or benzyl substituted thioamides $\mathbf{2 a - 2 f}$ afforded corresponding ethyl-2-methyl, phenyl or benzyl-substituted-4methylthiazole-5-carboxylate which on alkaline hydrolysis furnished corresponding 2-methyl, phenyl or benzylsubstituted-4-methylthiazole-5-carboxylic acids 3a-3f. The structures of acid $\mathbf{3 a}-\mathbf{3 f}$ were confirmed by spectral data. The IR spectrum of acid 3a displayed the broad absorption band at $2550-3400 \mathrm{~cm}^{-1}$ characteristic of - $\mathrm{COOH}$ group and a band at $1702 \mathrm{~cm}^{-1}$ due to stretching frequency of $\mathrm{C}=\mathrm{O}$.

The reaction of $\mathbf{3 a} \mathbf{a}-\mathbf{3 f}$ with $\mathrm{N}$-aryl or benzyl substituted piperazine $\mathbf{4 a}-\mathbf{b}$ in the presence of EDC-HOBt in DMF furnished the target compounds $\mathbf{5 a}-\mathbf{5 l}$ in good yields. The yields, melting points and molecular formula of synthesized compounds $\mathbf{5 a}-\mathbf{5 l}$ are listed in Table 1. The structures of synthesized compounds were characterized by IR, ${ }^{1} \mathrm{H}$
$\mathrm{NMR},{ }^{13} \mathrm{C} \mathrm{NMR}$, and mass spectroscopy and all the spectral data are in accordance with the assumed structures.

The IR spectrum of $\mathbf{5 d}$ showed the absorption band at $1699-1647 \mathrm{~cm}^{-1}$ and $1610-1590 \mathrm{~cm}^{-1}$ which indicated the presence of $\mathrm{O}=\mathrm{C}-\mathrm{N}$ (amide) and $\mathrm{C}=\mathrm{N}$ functionality respectively. The ${ }^{1} \mathrm{H}$ NMR spectrum of $\mathbf{5 d}$ revealed a singlet at $\delta$ 2.44 for thiazole- $\mathrm{CH}_{3}$ protons, two peaks at $\delta 3.13$ and 3.72 integrated for four protons of piperazine and a singlet at $\delta$ 4.24 integrated for two methylene protons of benzyl group. Seven aromatic protons resonated at $\delta 6.69-7.30$. Further the ${ }^{13} \mathrm{C}$ NMR spectrum of $\mathbf{5 d}$ revealed four signals in aliphatic region. The aromatic ipso carbon attached to fluorine appeared as doublet with ${ }^{1} J=244.4 \mathrm{~Hz}(\mathrm{C}-\mathrm{F})$. The ortho and the meta carbon atoms in the same ring also appeared as doublets with ${ }^{2} J=21.3 \mathrm{~Hz}_{\text {and }}^{3} J=8.0 \mathrm{~Hz}$. All other aromatic carbon and carbonyl carbon atoms appeared between $\delta$ 115.7 and 170.3. The structure was further confirmed by LC mass spectral data which showed peak at $464.1(\mathrm{M}+\mathrm{H})^{+}$.

\section{BIOLOGICAL ACTIVITY}

\section{Antimicrobial Activity}

The in vitro antibacterial activity was performed against Gram-positive bacteria including $S$. aureus, B. subtilis and Gram-negative bacteria including E. coli and $K$. pneumonia. The antifungal activity was screened against fungi including F. solani, C. lunata and A. niger. To evaluate the activity of the synthesized compounds, the zone of inhibition were determined. The in vitro antimicrobial screen- 
Scheme 1.
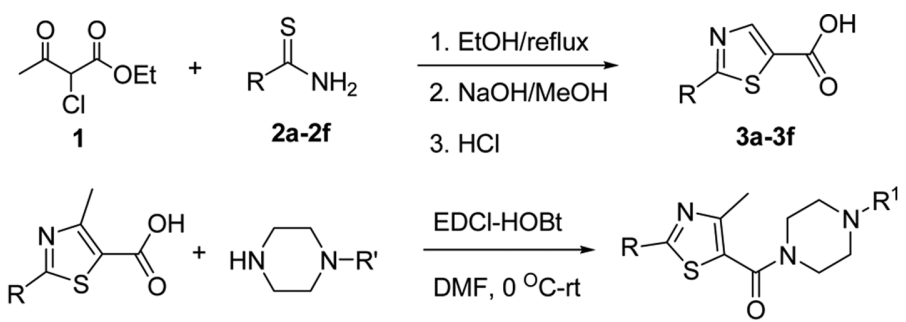

3a-3f $\quad 4 a-4 b$

Compound $\mathrm{R}$

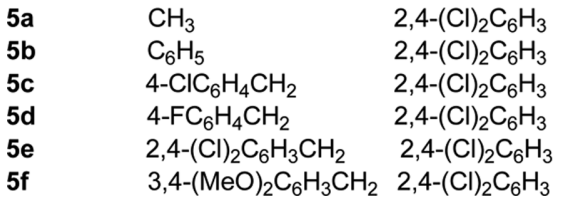

Compound
$5 \mathrm{~g}$
$5 \mathrm{~h}$
$5 \mathrm{i}$
$5 \mathrm{j}$
$5 \mathrm{k}$
$5 \mathrm{I}$

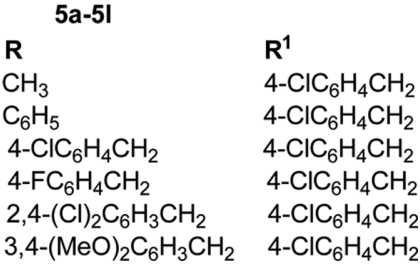

Table 1. Yield and physical data of compounds $\mathbf{5 a}-\mathbf{5 1}$

\begin{tabular}{llllll}
\hline Compound & $\mathrm{R}$ & $\mathrm{R}^{1}$ & $\mathrm{Mp}\left({ }^{\circ} \mathrm{C}\right)$ & Yield (\%) & Molecular Formula \\
\hline $\mathbf{5 a}$ & $\mathrm{CH}_{3}$ & $2,4-(\mathrm{Cl})_{2} \mathrm{C}_{6} \mathrm{H}_{3}$ & $82-84$ & 70 & $\mathrm{C}_{16} \mathrm{H}_{17} \mathrm{Cl}_{2} \mathrm{~N}_{3} \mathrm{OS}$ \\
$\mathbf{5 b}$ & $\mathrm{C}_{6} \mathrm{H}_{5}$ & $2,4-(\mathrm{Cl})_{2} \mathrm{C}_{6} \mathrm{H}_{3}$ & $136-138$ & 72 & $\mathrm{C}_{21} \mathrm{H}_{19} \mathrm{Cl}_{2} \mathrm{~N}_{3} \mathrm{OS}$ \\
$\mathbf{5 c}$ & $4-\mathrm{ClC}_{6} \mathrm{H}_{4} \mathrm{CH}_{2}$ & $2,4-(\mathrm{Cl})_{2} \mathrm{C}_{6} \mathrm{H}_{3}$ & $128-130$ & 70 & $\mathrm{C}_{22} \mathrm{H}_{20} \mathrm{Cl}_{3} \mathrm{~N}_{3} \mathrm{OS}$ \\
$\mathbf{5 d}$ & $4-\mathrm{OC}_{6} \mathrm{H}_{4} \mathrm{CH}_{2}$ & $2,4-(\mathrm{Cl})_{2} \mathrm{C}_{6} \mathrm{H}_{3}$ & $86-88$ & 70 & $\mathrm{C}_{22} \mathrm{H}_{20} \mathrm{Cl}_{2} \mathrm{FN}_{3} \mathrm{OS}$ \\
$\mathbf{5 e}$ & $2,4-(\mathrm{Cl})_{2} \mathrm{C}_{6} \mathrm{H}_{3} \mathrm{CH}_{2}$ & $2,4-(\mathrm{Cl})_{2} \mathrm{C}_{6} \mathrm{H}_{3}$ & $68-70$ & 65 & $\mathrm{C}_{22} \mathrm{H}_{19} \mathrm{Cl}_{4} \mathrm{~N}_{3} \mathrm{OS}$ \\
$\mathbf{5 f}$ & $3,4-(\mathrm{MeO})_{2} \mathrm{C}_{6} \mathrm{H}_{3} \mathrm{CH}_{2}$ & $2,4-(\mathrm{Cl})_{2} \mathrm{C}_{6} \mathrm{H}_{3}$ & $89-91$ & 74 & $\mathrm{C}_{24} \mathrm{H}_{25} \mathrm{Cl}_{2} \mathrm{~N}_{3} \mathrm{O}_{3} \mathrm{~S}$ \\
$\mathbf{5 g}$ & $\mathrm{CH}_{3}$ & $4-\mathrm{ClC}_{6} \mathrm{H}_{4} \mathrm{CH}_{2}$ & $78-79$ & 75 & $\mathrm{C}_{17} \mathrm{H}_{20} \mathrm{ClN}_{3} \mathrm{OS}$ \\
$\mathbf{5 h}$ & $\mathrm{C}_{6} \mathrm{H}_{5}$ & $4-\mathrm{ClC}_{6} \mathrm{H}_{4} \mathrm{CH}_{2}$ & $83-85$ & 65 & $\mathrm{C}_{22} \mathrm{H}_{22} \mathrm{ClN}_{3} \mathrm{OS}$ \\
$\mathbf{5 i}$ & $4-\mathrm{ClC}_{6} \mathrm{H}_{4} \mathrm{CH}_{2}$ & $4-\mathrm{ClC}_{6} \mathrm{H}_{4} \mathrm{CH}_{2}$ & $86-88$ & 65 & $\mathrm{C}_{23} \mathrm{H}_{23} \mathrm{Cl}_{2} \mathrm{~N}_{3} \mathrm{OS}$ \\
$\mathbf{5 j}$ & $4-\mathrm{FC}_{6} \mathrm{H}_{4} \mathrm{CH}_{2}$ & $4-\mathrm{ClC}_{6} \mathrm{H}_{4} \mathrm{CH}_{2}$ & $160-162$ & 70 & $\mathrm{C}_{23} \mathrm{H}_{23} \mathrm{ClFN}_{3} \mathrm{OS}$ \\
$\mathbf{5 k}$ & $2,4-(\mathrm{Cl})_{2} \mathrm{C}_{6} \mathrm{H}_{3} \mathrm{CH}_{2}$ & $4-\mathrm{ClC}_{6} \mathrm{H}_{4} \mathrm{CH}_{2}$ & $132-135$ & 75 & $\mathrm{C}_{23} \mathrm{H}_{22} \mathrm{Cl}_{3} \mathrm{~N}_{3} \mathrm{OS}$ \\
$\mathbf{5 1}$ & $3,4-(\mathrm{MeO})_{2} \mathrm{C}_{6} \mathrm{H}_{3} \mathrm{CH}_{2}$ & $4-\mathrm{ClC}_{6} \mathrm{H}_{4} \mathrm{CH}_{2}$ & $78-80$ & 60 & $\mathrm{C}_{25} \mathrm{H}_{28} \mathrm{ClN}_{3} \mathrm{O}_{3} \mathrm{~S}$ \\
\hline
\end{tabular}

Table 2. Antibacterial screening results of the compounds $\mathbf{5 a}-\mathbf{5 l}$ (Zone diameter of growth inhibition in $\mathrm{mm}$ )

\begin{tabular}{|c|c|c|c|c|c|c|c|c|c|}
\hline \multirow{2}{*}{ Compd. } & \multicolumn{3}{|c|}{ S. aureous } & \multicolumn{2}{|c|}{ B. subtilis } & \multicolumn{2}{|c|}{ E. coli } & \multicolumn{2}{|c|}{ K. pneumonia } \\
\hline & Conc. $\mu \mathrm{g} / \mathrm{ml}$ & 100 & 200 & 100 & 200 & 100 & 200 & 100 & 200 \\
\hline $5 \mathbf{5 a}$ & & 26 & 30 & 24 & 27 & 25 & 29 & 23 & 26 \\
\hline $5 \mathbf{b}$ & & 26 & 29 & 29 & 31 & 31 & 34 & 28 & 31 \\
\hline $5 c$ & & 24 & 27 & 27 & 29 & 28 & 29 & 26 & 28 \\
\hline $5 d$ & & 10 & 12 & 10 & 13 & 9 & 12 & 8 & 10 \\
\hline $5 e$ & & 33 & 36 & 37 & 39 & 39 & 44 & 34 & 37 \\
\hline $5 \mathrm{~g}$ & & 36 & 39 & 34 & 37 & 31 & 34 & 29 & 33 \\
\hline $5 \mathrm{~h}$ & & 31 & 34 & 32 & 35 & 37 & 38 & 34 & 36 \\
\hline $5 \mathbf{i}$ & & 9 & 12 & 8 & 13 & 7 & 12 & 6 & 10 \\
\hline $5 \mathbf{j}$ & & 28 & 31 & 28 & 32 & 27 & 33 & 26 & 29 \\
\hline $5 \mathbf{k}$ & & 38 & 43 & 37 & 41 & 36 & 40 & 37 & 39 \\
\hline 51 & & 7 & 9 & 8 & 9 & 7 & 9 & 6 & 8 \\
\hline Ciprofloxacin & & 34 & 38 & 36 & 42 & 40 & 45 & 37 & 42 \\
\hline Chloramphenicol & & 35 & 39 & 38 & 41 & 40 & 44 & 42 & 45 \\
\hline
\end{tabular}

ing results of tested compounds are listed in Table 2 and 3.

Most of the synthesized compounds (except compounds 5d, 5i and 5l) exhibited moderate to excellent antimicro- bial activities. It is noteworthy that compounds $\mathbf{5 e}$ and $\mathbf{5 k}$ $\left(\mathrm{R}=2,4-(\mathrm{Cl})_{2} \mathrm{C}_{6} \mathrm{H}_{3} \mathrm{CH}_{2}-, \mathrm{R}^{1}=2,4-\left(\mathrm{Cl}_{2} \mathrm{C}_{6} \mathrm{H}_{3}-\right.\right.$ and $4-\mathrm{ClC}_{6} \mathrm{H}_{4} \mathrm{CH}_{2}$, respectively), compound $5 \mathrm{~g}\left(\mathrm{R}=\mathrm{CH}_{3}-, \mathrm{R}^{1}=4-\mathrm{ClC}_{6} \mathrm{H}_{4} \mathrm{CH}_{2}-\right)$ 
Table 3. Antifungal screening results of the compounds $\mathbf{5 a}-\mathbf{5 1}$ (Zone diameter of growth inhibition in $\mathbf{m m}$ )

\begin{tabular}{|c|c|c|c|c|c|c|c|}
\hline \multirow{2}{*}{ Compd. } & \multicolumn{3}{|c|}{ F. solani } & \multicolumn{2}{|c|}{ C. lunata } & \multicolumn{2}{|c|}{ A. niger } \\
\hline & Conc. $\mu \mathrm{g} / \mathrm{ml}$ & 100 & 200 & 100 & 200 & 100 & 200 \\
\hline 5 & & 16 & 19 & 17 & 20 & 15 & 18 \\
\hline $5 b$ & & 19 & 21 & 22 & 24 & 17 & 21 \\
\hline $5 c$ & & 24 & 27 & 23 & 25 & 20 & 24 \\
\hline $5 d$ & & 29 & 32 & 27 & 31 & 22 & 24 \\
\hline $5 e$ & & 16 & 19 & 17 & 19 & 15 & 16 \\
\hline $5 g$ & & 14 & 19 & 14 & 18 & 13 & 17 \\
\hline $5 \mathrm{~h}$ & & 17 & 20 & 18 & 22 & 16 & 21 \\
\hline $5 i$ & & 17 & 19 & 18 & 23 & 18 & 21 \\
\hline $5 j$ & & 21 & 23 & 18 & 20 & 17 & 21 \\
\hline $5 \mathbf{k}$ & & 16 & 20 & 15 & 17 & 16 & 19 \\
\hline 51 & & 24 & 26 & 24 & 27 & 18 & 20 \\
\hline Ketoconazole & & 38 & 42 & 38 & 42 & 38 & 42 \\
\hline
\end{tabular}

and $\mathbf{5 h}\left(\mathrm{R}=\mathrm{C}_{6} \mathrm{H}_{5}-, \mathrm{R}^{1}=4-\mathrm{ClC}_{6} \mathrm{H}_{4} \mathrm{CH}_{2}-\right)$ showed excellent activity against all bacterial strain as compared to standard drugs. Even compounds $\mathbf{5 a}, \mathbf{5 b}, \mathbf{5} \mathbf{c}$ and $\mathbf{5 j}$ showed good antibacterial activities against most of the strains.

The result of antifungal activity revealed that all the tested compounds show moderate to good antifungal activity as compared to the standard drug ketoconazole. Compound 5c $\left(\mathrm{R}=4-\mathrm{ClC}_{6} \mathrm{H}_{4} \mathrm{CH}_{2}-, \mathrm{R}^{1}=2,4-(\mathrm{Cl})_{2} \mathrm{C}_{6} \mathrm{H}_{3}-\right), \mathbf{5 d}(\mathrm{R}=4-$ $\left.\mathrm{FC}_{6} \mathrm{H}_{4} \mathrm{CH}_{2}-, \mathrm{R}^{1}=2,4-(\mathrm{Cl})_{2} \mathrm{C}_{6} \mathrm{H}_{3}-\right)$ and $5 \mathrm{l}\left(\mathrm{R}=3,4-(\mathrm{MeO})_{2}\right.$ $\mathrm{C}_{6} \mathrm{H}_{3} \mathrm{CH}_{2}-, \mathrm{R}^{1}=4-\mathrm{ClC}_{6} \mathrm{H}_{4} \mathrm{CH}_{2}-$ ) exhibited good activities against all the fungal species. However compounds $\mathbf{5 d}, \mathbf{5 i}$ and $\mathbf{5 l}$ were inactive against bacterial strain but showed good antifungal activity.

Thus, it is concluded that compounds with $\mathrm{R}=\mathrm{CH}_{3}-, \mathrm{C}_{6} \mathrm{H}_{5}-$ and 2,4-diClC $\mathrm{H}_{3} \mathrm{CH}_{2}$ - show good to excellent antibacterial activity.

\section{EXPERIMENTAL}

Melting points were determined in an open capillary on Veego melting point apparatus and are uncorrected. The purity of the compounds was checked on silica gel-G plates. The compounds 5a-l was purified on silica gel (100-200) column chromatography using ethyl acetate: hexane (2:8) as eluent. Infrared spectra $\left(\mathrm{cm}^{-1}\right)$ were recorded in $\mathrm{KBr}$ on a Shimadzu Model FTIR-435 spectrophotometer. ${ }^{1} \mathrm{H}$ NMR and ${ }^{13} \mathrm{C}$ NMR spectra were recorded in $\mathrm{CDCl}_{3}$ or DMSO- $d_{6}$ solution on a Varian Mercury YH-300 spectrometer operating at $300 \mathrm{MHz}$ for ${ }^{1} \mathrm{H}$ and $75 \mathrm{MHz}$ for ${ }^{13} \mathrm{C}$. Chemical shifts are expressed relative to tetramethylsilane (TMS) and were reported as $\delta$ (ppm). Mass spectral (MS) measurements were made on a Jeol-JMS-DX 303 mass spectrometer. The isotopic peak at $\mathrm{M}+2$ was observed in the mass spectrum of all the compounds due to $\mathrm{S}, \mathrm{Br}$ and/or $\mathrm{Cl}$.

\section{General Procedure}

2-Methyl, phenyl or benzyl substituted-4-methylthiazole-5-carboxylic acid (3a-3f)

Mixture of 2-chloro-3-oxo ethyl butanoate $(0.05 \mathrm{~mol})$ and substituted thioamides $(0.055 \mathrm{~mol})$ in methanol $(50 \mathrm{~mL})$ was refluxed for 5-6 hours. After completion of the reaction, as monitored on TLC, $2 \mathrm{~N} \mathrm{NaOH}(20 \mathrm{~mL})$ was added and refluxed further for 4 hours. Methanol was distilled off and the mixture was acidified with $4 \mathrm{~N} \mathrm{HCl}$ to $\mathrm{pH} 2$. The precipitated product was filtered, washed with water and recrystallized from aqueous ethanol.

(2-methyl, phenyl or benzyl-substituted-4-methylthiazol5-yl)(4-phenyl or benzyl substituted piperazin-1-yl)methanone $(5 a-5 l)$

A mixture of 2-substituted-4-methylthiazole-5-carboxylic acid (3a-f) (1 mmol), DIPEA (0.35 mL, $2 \mathrm{mmol}), \mathrm{HOBt}$ $(0.14 \mathrm{~g}, 1 \mathrm{mmol})$ in DMF $(10 \mathrm{~mL})$ was cooled to $0{ }^{\circ} \mathrm{C}$. To this $\mathrm{N}$-aryl or benzyl piperazine $(\mathbf{4 a}-\mathbf{b})(1 \mathrm{mmol})$ was added followed by $\mathrm{EDC} . \mathrm{HCl}(0.19 \mathrm{~g}, 1 \mathrm{mmol})$ at $0{ }^{\circ} \mathrm{C}$ and stirred overnight at room temperature. The reaction was quenched with water and the product was filtered, washed with water and purified by column chromatography using ethyl acetate:hexane (2:8).

(4-(2,4-Dichlorophenyl)piperazin-1-yl)(2,4-dimethylthiazol-5-yl)methanone (5a)

IR (KBr): 3056, 2971, 2911, 2802, 1631, 1542, 1480, 1417, 1251, 1130, 1006, 934, 830, $822 \mathrm{~cm}^{-1} ;{ }^{1} \mathrm{H} \mathrm{NMR}\left(\mathrm{CDCl}_{3}\right.$, $300 \mathrm{MHz}): 2.42$ (s, 3H), 2.68 (s, 3H), $3.18(\mathrm{~s}, 4 \mathrm{H}), 3.76(\mathrm{~s}$, $4 \mathrm{H}), 6.72(\mathrm{~m}, 1 \mathrm{H}), 6.95(\mathrm{~s}, 1 \mathrm{H}), 7.24-7.45(\mathrm{~m}, 1 \mathrm{H}) ;{ }^{13} \mathrm{C}$ 
$\operatorname{NMR}\left(\mathrm{CDCl}_{3}, 75 \mathrm{MHz}\right): 15.0,20.1,48.1,49.3,115.4,121.6$, $126.8,127.0,128.9,132.5,149.3,151.7,164.2,168.5$ LCMS: $370.0(\mathrm{M}+\mathrm{H})^{+}$. Anal. calcd. for $\mathrm{C}_{16} \mathrm{H}_{17} \mathrm{Cl}_{2} \mathrm{~N}_{3} \mathrm{OS}$ : C, 51.90; H, 4.63; N, 11.35. Found: C, 51.79; H, 4.51; N, 11.22 .

(4-(2,4-Dichlorophenyl)piperazin-1-yl)(4-methyl-2-phenylthiazol-5-yl)methanone (5b)

IR (KBr): 2991, 2891, 2850, 1616, 1541, 1438, 1365, $1242,1151,1010,947,850,813 \mathrm{~cm}^{-1} ;{ }^{1} \mathrm{H} \mathrm{NMR}\left(\mathrm{CDCl}_{3}\right.$, $300 \mathrm{MHz}): 2.44$ (s, 3H), 3.11 (s, 4H), 3.71 (s, 4H), 6.66 $(\mathrm{dd}, J=3$ and $9 \mathrm{~Hz}, 1 \mathrm{H}), 6.88,(\mathrm{~d}, J=3 \mathrm{~Hz}, 1 \mathrm{H}), 7.21(\mathrm{~d}, J$ $=9 \mathrm{~Hz}, 1 \mathrm{H}), 7.35-7.37(\mathrm{~m}, 3 \mathrm{H}), 7.81-7.85(\mathrm{~m}, 2 \mathrm{H}) ;{ }^{13} \mathrm{C}$ $\operatorname{NMR}\left(\mathrm{CDCl}_{3}, 75 \mathrm{MHz}\right): 16.6,49.1,115.9,117.9,123.3$, 123.7, 126.5, 129.0, 130.5, 130.6, 132.7, 132.9, 150.0, 153.1, 162.6, 167.9; LCMS: $432.1(\mathrm{M}+\mathrm{H})^{+}$. Anal. calcd. for $\mathrm{C}_{21} \mathrm{H}_{19} \mathrm{Cl}_{2} \mathrm{~N}_{3} \mathrm{OS}$ : $\mathrm{C}$, 58.34; $\mathrm{H}, 4.43$; N, 9.72. Found: $\mathrm{C}$, 58.26; H, 4.37; N, 9.59.

(2-(4-Chlorobenzyl)-4-methylthiazol-5-yl)(4-(2,4-dichlorophenyl)piperazin-1-yl)methanone (5c)

IR (KBr): 3059, 2930, 2879, 2823, 1631, 1475, 1427, 1365, 1271, 1240, 1157, 1089, 1016, 956, $873 \mathrm{~cm}^{-1}$; ${ }^{1} \mathrm{H}$ NMR $\left(\mathrm{CDCl}_{3}, 300 \mathrm{MHz}\right): 2.41$ (s, 3H), $3.16(\mathrm{~s}, 4 \mathrm{H}), 3.61$ (s, 4H), $3.84(\mathrm{~s}, 2 \mathrm{H}), 6.75(\mathrm{dd}, J=2.4$ and $8.4 \mathrm{~Hz}, 1 \mathrm{H}), 7.00(\mathrm{~d}, J=$ $2.4 \mathrm{~Hz}, 1 \mathrm{H}), 7.30$ (d, $J=8.4 \mathrm{~Hz}, 1 \mathrm{H}), 7.37-7.42(\mathrm{~m}, 4 \mathrm{H})$;

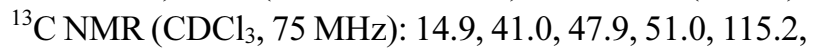
117.8, 125.0, 125.5, 128.1, 128.8, 130.6, 131.4, 131.6, 134.3, 148.9, 152.0, 164.5, 169.1; LCMS: $480.0(\mathrm{M}+\mathrm{H})^{+}$. Anal. calcd. for $\mathrm{C}_{22} \mathrm{H}_{20} \mathrm{Cl}_{3} \mathrm{~N}_{3} \mathrm{OS}$ : C, 54.95; H, 4.19; N, 8.74. Found: C, 54.88; H, 4.11; N, 8.63.

(2-(4-Fluorobenzyl)-4-methylthiazol-5-yl)(4-(2,4-dichlorophenyl)piperazin-1-yl)methanone (5d)

IR (KBr): 3076, 2908, 2831, 1947, 1506, 1435, 1348, 1246, $1138,1006,852,763 \mathrm{~cm}^{-1}$; ${ }^{1} \mathrm{H}$ NMR $\left(\mathrm{CDCl}_{3}, 300 \mathrm{MHz}\right)$ : $2.44(\mathrm{~s}, 3 \mathrm{H}), 3.13(\mathrm{~s}, 4 \mathrm{H}), 3.72(\mathrm{~s}, 4 \mathrm{H}), 4.24(\mathrm{~s}, 2 \mathrm{H}), 6.71$ (dd, $J=2.75$ and $9 \mathrm{~Hz}, 1 \mathrm{H}), 6.83,(\mathrm{~d}, J=2.70 \mathrm{~Hz}, 1 \mathrm{H}), 7.01$ (t, $J=8.7 \mathrm{~Hz}, 2 \mathrm{H}), 7.24-7.30(\mathrm{~m}, 3 \mathrm{H}) ;{ }^{13} \mathrm{C} \mathrm{NMR}\left(\mathrm{CDCl}_{3}, 75\right.$ $\mathrm{MHz})$ : 16.2, 38.4, 48.6, 48.8, 115.4, 117.6, 122.8, 124.0, 130.3, 130.5, 132.5, 132.6, 132.7, 149.9, 151.7, 161.5, 162.4, 170.3; LCMS: $464.1(\mathrm{M}+\mathrm{H})^{+}$. Anal. calcd. for $\mathrm{C}_{22} \mathrm{H}_{20} \mathrm{Cl}_{2} \mathrm{FN}_{3} \mathrm{OS}$ : C, 56.90; H, 4.34; N, 9.05. Found: C, 56.80; H, 4.23; N, 8.94.

(2-(2,4-Dichlorobenzyl)-4-methylthiazol-5-yl)(4-(2,4dichlorophenyl)piperazin-1-yl)methanone (5e)

IR (KBr): 3084, 2928, 2833, 1631, 1558, 1487, 1242, 997, 954, 856, $788 \mathrm{~cm}^{-1}$; ${ }^{1} \mathrm{H}$ NMR ( $\left.\mathrm{CDCl}_{3}, 300 \mathrm{MHz}\right): 2.36$ (s, $3 \mathrm{H}), 3.06(\mathrm{~s}, 4 \mathrm{H}), 3.64(\mathrm{~s}, 4 \mathrm{H}), 4.29$ (s, 2H), 6.64 (dd, $J=3$ and $9 \mathrm{~Hz}, 1 \mathrm{H}), 6.86,(\mathrm{~d}, J=3 \mathrm{~Hz}, 1 \mathrm{H}), 7.13-7.23(\mathrm{~m}, 3 \mathrm{H})$, $7.33(\mathrm{~d}, J=2.1 \mathrm{~Hz}, 1 \mathrm{H}) ;{ }^{13} \mathrm{C} \mathrm{NMR}\left(\mathrm{CDCl}_{3}, 75 \mathrm{MHz}\right)$ : 16.4, 29.6, 36.4, 49.0, 115.4, 117.9, 123.2, 124.2, 127.5, $129.5,130.5,131.9,132.8,133.4,134.0,134.7,150.0$, 151.9, 162.5, 168.1; LCMS: $514.0(\mathrm{M}+\mathrm{H})^{+}$. Anal. calcd. for $\mathrm{C}_{22} \mathrm{H}_{19} \mathrm{Cl}_{4} \mathrm{~N}_{3} \mathrm{OS}: \mathrm{C}, 51.28 ; \mathrm{H}, 3.72 ; \mathrm{N}, 8.15$. Found: $\mathrm{C}$, $51.20 ; \mathrm{H}, 3.60 ; \mathrm{N}, 8.04$.

(2-(3,4-Dimethoxybenzyl)-4-methylthiazol-5-yl)(4-(2,4dichlorophenyl)piperazin-1-yl)methanone (5f)

IR (KBr): 3031, 2965, 2910, 2824, 1631, 1524, 1418, 1315, 1251, 1140, 1089, 1006, 750, $819 \mathrm{~cm}^{-1}$; ${ }^{1} \mathrm{H}$ NMR $\left(\mathrm{CDCl}_{3}, 300 \mathrm{MHz}\right): 2.43$ (s, 3H), 3.14 (s, 4H), 3.73 (s, 4H), $3.86(\mathrm{~s}, 3 \mathrm{H}), 3.87$ (s, 3H), 4.19 (s, $2 \mathrm{H}), 6.72$ (dd, $J=3$ and $9 \mathrm{~Hz}, 1 \mathrm{H}), 6.80-6.87,(\mathrm{~m}, 3 \mathrm{H}), 6.94(\mathrm{~d}, J=3 \mathrm{~Hz}, 1 \mathrm{H}), 7.28$ (d, $J=9 \mathrm{~Hz} 1 \mathrm{H}) ;{ }^{13} \mathrm{C}$ NMR $\left(\mathrm{CDCl}_{3}, 75 \mathrm{MHz}\right): 15.4,39.2$, 46.4, 51.1, 56.2, 56.3, 115.2, 117.0, 117.6, 119.1, 123.4, 125.2, 126.0, 128.1, 129.5, 131.5, 145.8, 148.4, 149.7, 152.2, 163.1, 168.2; LCMS: $506.1(\mathrm{M}+\mathrm{H})^{+}$. Anal. calcd. for $\mathrm{C}_{24} \mathrm{H}_{25} \mathrm{Cl}_{2} \mathrm{~N}_{3} \mathrm{O}_{3} \mathrm{~S}$ : C, 56.92; H, 4.98; N, 8.30. Found: C, 56.78; H, 4.86; N, 8.19.

(4-(4-Chlorobenzyl)piperazin-1-yl)(2,4-dimethylthiazol-5yl)methanone (5g)

IR (KBr) 3060, 2976, 2908, 2808, 1639, 1546, 1487, 1429, 1265, 1138, 1006, 935, 831, $756 \mathrm{~cm}^{-1}$; ${ }^{1} \mathrm{H}$ NMR: 2.37 (s, $7 \mathrm{H}), 2.60(\mathrm{~s}, 3 \mathrm{H}), 3.59(\mathrm{~s}, 4 \mathrm{H}), 4.23(\mathrm{~s}, 2 \mathrm{H}), 7.15-7.34(\mathrm{~m}$, 4H); ${ }^{13} \mathrm{C}$ NMR: $15.1,20.2,48.9,59.1,65.3,116.0,128.9$, 130.5, 132.9, 135.1, 152.0, 164.5, 168.3; LCMS: 350.1 $(\mathrm{M}+\mathrm{H})^{+}$. Anal. calcd. for $\mathrm{C}_{17} \mathrm{H}_{20} \mathrm{ClN}_{3} \mathrm{OS}$ : C, 58.36; H, 5.76; N, 12.01. Found: C, 58.23; H, 5.67; N, 11.89.

(4-(4-Chlorobenzyl)piperazin-1-yl)(4-methyl-2-phenylthiazol-5-yl)methanone (5h)

IR (KBr): 3065, 2956, 2900, 2815, 1630, 1484, 1440, $1321,1250,1005,865,815 \mathrm{~cm}^{-1}$; ${ }^{1} \mathrm{HNMR}\left(\mathrm{CDCl}_{3}, 300 \mathrm{MHz}\right)$ : $2.26(\mathrm{~s}, 4 \mathrm{H}), 2.35(\mathrm{~s}, 3 \mathrm{H}), 3.49(\mathrm{~s}, 4 \mathrm{H}), 4.09(\mathrm{~s}, 2 \mathrm{H}), 7.01-$ $7.24(\mathrm{~m}, 7 \mathrm{H}), 7.74-7.77(\mathrm{~m}, 2 \mathrm{H}) ;{ }^{13} \mathrm{C} \mathrm{NMR}\left(\mathrm{CDCl}_{3}, 75\right.$ $\mathrm{MHz})$ : 18.1, 51.5, 74.7, 77.2, 119.4, 126.2, 127.4, 128.4, 128.7, 130.2, 133.6, 140.1, 140.6, 152.2, 162.2, 167.6; LCMS: $412.1(\mathrm{M}+\mathrm{H})^{+}$. Anal. calcd. for $\mathrm{C}_{22} \mathrm{H}_{22} \mathrm{ClN}_{3} \mathrm{OS}$ : C, 64.14; H, 5.38; N, 10.20. Found: C, 64.08; H, 5.31; N, 10.09.

(2-(4-Chlorobenzyl)-4-methylthiazol-5-yl)(4-(4-chlorobenzyl)piperazin-1-yl)methanone (5i)

IR (KBr): 3068, 2949, 2912, 2809, 1628, 1504, 1478, 1324, 1248, 1006, 860, $809 \mathrm{~cm}^{-1} ;{ }^{1} \mathrm{H}$ NMR $\left(\mathrm{CDCl}_{3}, 300\right.$ MHz): 2.32 (s, 3H), 2.60 (s, 4H), 3.32 (s, 4H), 4.21 (s, 2H), $4.23(\mathrm{~s}, 2 \mathrm{H}), 7.15-7.36(\mathrm{~m}, 8 \mathrm{H}) ;{ }^{13} \mathrm{C} \mathrm{NMR}\left(\mathrm{CDCl}_{3}, 75 \mathrm{MHz}\right)$ : 14.2, 47.7, 51.2, 61.2, 127.2, 127.5, 128.3, 128.5, 128.9, 129.7, 
131.2, 132.6, 133.8, 135.5, 140.4, 153.2, 168.9; LCMS: 460.1 $(\mathrm{M}+\mathrm{H})^{+}$. Anal. calcd. for $\mathrm{C}_{23} \mathrm{H}_{23} \mathrm{Cl}_{2} \mathrm{~N}_{3} \mathrm{OS}: \mathrm{C}, 60.00 ; \mathrm{H}, 5.04$ N, 9.13. Found: C, 59.88; H, 5.00; N, 9.02.

\section{(4-(4-Chlorobenzyl)piperazin-1-yl)(2-(4-fluorobenzyl)-4 methylthiazol-5-yl)methanone (5j)}

IR (KBr): 3051, 2908, 2823, 1629, 1487, 1305, 1240, $1132,1006,846,746 \mathrm{~cm}^{-1}$; ${ }^{1} \mathrm{H} \mathrm{NMR}\left(\mathrm{CDCl}_{3}, 300 \mathrm{MHz}\right)$ : $2.41(\mathrm{~s}, 4 \mathrm{H}), 2.44(\mathrm{~s}, 3 \mathrm{H}), 3.62(\mathrm{~s}, 4 \mathrm{H}), 4.24(\mathrm{~s}, 2 \mathrm{H}), 4.26$ (s, 2H), $7.03(\mathrm{t}, J=8.4 \mathrm{~Hz}, 2 \mathrm{H}), 7.20-7.40(\mathrm{~m}, 6 \mathrm{H}) ;{ }^{13} \mathrm{C}$ NMR $\left(\mathrm{CDCl}_{3}, 75 \mathrm{MHz}\right): 16.2,38.5,51.6,60.1,75.0,115.5$, $124.5,127.5,128.6,128.9,130.4,132.8,141.0,151.3$, 160.2, 163.5, 170.0; LCMS: $444.1(\mathrm{M}+\mathrm{H})^{+}$. Anal. calcd. for $\mathrm{C}_{23} \mathrm{H}_{23} \mathrm{ClFN}_{3} \mathrm{OS}$ : C, 62.22; $\mathrm{H}, 5.22 ; \mathrm{N}, 9.46$. Found: $\mathrm{C}$, 62.09; H, 5.17; N, 9.33 .

(2-(2,4-Dichlorobenzyl)-4-methylthiazol-5-yl)(4-(4-chlorobenzyl)piperazin-1-yl)methanone (5k)

IR (KBr): 3070, 2970, 2910, 2808, 1627, 1545, 1477, 1440, 1311, 1257, 1099, 1006, 864, $813 \mathrm{~cm}^{-1}$; ${ }^{1} \mathrm{H} \mathrm{NMR}\left(\mathrm{CDCl}_{3}\right.$, $300 \mathrm{MHz}): 2.30$ (s, 7H), 3.49 (s, 4H), 4.12 (s, 2H), 4.23 (s, 2H), 7.06-7.29 (m, 7H); $\left.{ }^{13} \mathrm{C} \mathrm{NMR} \mathrm{(} \mathrm{CDCl}_{3}, 75 \mathrm{MHz}\right): 16.2$, 36.3, 51.6, 74.9, 124.5, 127.3, 127.5, 128.6, 128.9, 129.4, 131.8, 133.5, 133.8, 134.6, 140.4, 141.2, 151.3, 162.2, 167.7; LCMS: $494.1(\mathrm{M}+\mathrm{H})^{+}$. Anal. calcd. for $\mathrm{C}_{23} \mathrm{H}_{22} \mathrm{Cl}_{3} \mathrm{~N}_{3} \mathrm{OS}$ : C, 55.82; H, 4.48; N, 8.49. Found: C, 55.69; H, 4.36; N, 8.35.

(2-(3,4-Dimethoxybenzyl)-4-methylthiazol-5-yl)(4-(4chlorobenzyl)piperazin-1-yl)methanone (5l)

IR (KBr): 3024, 2974, 2918, 2810, 1626, 1533, 1429 , 1317, 1249, 1141, 1095, 1004, 754, $709 \mathrm{~cm}^{-1} ;{ }^{1} \mathrm{H}$ NMR $\left(\mathrm{CDCl}_{3}, 300 \mathrm{MHz}\right): 2.37$ (s, 4H), $2.62(\mathrm{~s}, 3 \mathrm{H}), 3.58(\mathrm{~s}, 4 \mathrm{H})$, $3.83(\mathrm{~s}, 6 \mathrm{H}), 4.17(\mathrm{~s}, 2 \mathrm{H}), 4.21(\mathrm{~s}, 2 \mathrm{H}), 6.81(\mathrm{t}, J=8.4 \mathrm{~Hz}$, 2H), 7.15-7.35 (m, 5H); $\left.{ }^{13} \mathrm{C} \mathrm{NMR} \mathrm{(} \mathrm{CDCl}_{3}, 75 \mathrm{MHz}\right): 15.2$, $39.8,48.0,55.3,56.2,56.3,64.3,115.1,116.8,117.5,123.3$, $128.8,129.7,130.5,132.9,133.6,146.0,148.6,152.1,163.1$, 168.2; LCMS: $485.2(\mathrm{M}+\mathrm{H})^{+}$. Anal. calcd. for $\mathrm{C}_{25} \mathrm{H}_{28} \mathrm{ClN}_{3} \mathrm{O}_{3} \mathrm{~S}$ : C, 61.78; H, 5.81; N, 8.65. Found: C, 61.64; H, 5.70; N, 8.49.

\section{Antimicrobial Activity}

The synthesized compounds $\mathbf{5 a}-\mathbf{5 l}$ were screened for their in vitro antimicrobial activity against the standard strains B. subtilis, S. aureus (Gram-positive) and E. coli, $K$. pneumonia (Gram-negative) by the disk diffusion method. ${ }^{17,18}$ Disks measuring $6 \mathrm{~mm}$ in diameter were punched from whatman no. 1 filter paper. Batches of 100 disks were dispensed to each screw-caped bottle and sterilized by dry heat at $145^{\circ} \mathrm{C}$ for one hour. The test compounds were prepared with $100 \mu \mathrm{g} / \mathrm{mL}$ and $200 \mu \mathrm{g} / \mathrm{mL}$ concentration in dimethyl sulfoxide (DMSO). Disks of each concentration were placed in nutrient agar medium inoculated with fresh bacteria strains separately. Ciprofloxacin and Chloramphenicol was used as positive controls and DMSO was used as negative control. The incubation was carried out at $37^{\circ} \mathrm{C}$ for $24 \mathrm{~h}$. The diameter of the zone of growth inhibition around each well was measured after incubation using vernier caliper.

The compounds were screened for their antifungal activity against $F$. solani, $C$. lunata and $A$. niger in DMSO by disc diffusion method under standard conditions using Sabourad Dextrose Agar medium as described by NCCLS. ${ }^{19}$ Sterile filter paper discs (6 mm diameter) containing specific amount of anti fungal agent (100 $\mu \mathrm{g}$ for the synthesized compounds) were placed on the surface of an agar plate inoculated with the standardized suspension of microorganisms tested. The plates were incubated at $37^{\circ} \mathrm{C}$ for 2 days for evaluating antifungal activity. The diameters of inhibition zones (in $\mathrm{mm}$ ) were measured. Ketoconazole was used as positive control.

\section{CONCLUSION}

In summary, we have synthesized a series of novel (2methyl, phenyl or benzyl-substituted-4-methylthiazol-5yl)(4-phenyl or benzyl substituted piperazin-1-yl)methanone $(\mathbf{5 a}-\mathbf{5 l})$ and screened for their antimicrobial activity. The in vitro antimicrobial results revealed that the compounds $\mathbf{5 e}, \mathbf{5 g}, \mathbf{5 h}$ and $\mathbf{5 k}$ show significant antibacterial activity while compounds $\mathbf{5 a}, \mathbf{5 b}, \mathbf{5} \mathbf{c}$ and $\mathbf{5 j}$ showed good antibacterial activities. All the tested compounds showed moderate to good antifungal activity. The antimicrobial activity results make them interesting lead molecules for further synthetic and biological evaluation. Further studies are in progress to acquire more information regarding structure activity relationship.

Acknowledgments. The authors are thankful to University Grant Commission, New Delhi for financial support. Garware Research Center, University of Pune and IIT Mumbai, India is gratefully acknowledged for providing spectral analysis facilities. And the publication cost of this paper was supported by the Korean Chemical Society.

\section{REFERENCES}

1. Hutchinson, I.; Jennings, S. A.; Vishnuvajjala, B. R.; Westwell, A. D.; Stevens, M. F. G. J. Med. Chem. 2002, 45,744 .

2. Nicolaou, K. C.; Roschanger, F.; Vourloumis, D. Angew. 
Chem. Int. Ed. 1998, 37, 2014.

3. Ojika, M.; Suzuki, Y.; Tsukamoto, A.; Sakagami, Y.; Fudou, R.; Yoshimura, T.; Yamanaka, S. J. Antibiot. 1998, 51, 275.

4. Suzuki, Y.; Ojika, M.; Sakagami, Y.; Fudou, R.; Yamanaka, S. Tetrahedron 1998, 54, 11399.

5. EI-Subbagh, H. I.; Al-Obaid, A.M. Eur. J. Med. Chem. 1996, 31, 1017.

6. Zhang, C.; Zink, D. L.; Ushio, M.; Burgess, B.; Onishi, R.; Masurekar, P.; Barrett, J. F.; Singh, S. B. Bioorg. Med. Chem. 2008, 16, 8818 .

7. Kalkhambkar, R. G.; Kulkarni, G. M.; Shivkumar, H.; Rao, N. R. Eur. J. Med. Chem. 2007, 42, 1272.

8. Franklin, P. X.; Pillai, A. D.; Rathod, P. D.; Yerande, S.; Nivsarkar, M.; Padh, K. K.; Sudarsanam, V. Eur. J. Med. Chem. 2008, 43, 129.

9. Zitouni, G. T.; Ozdemir, A.; Kaplancikli, Z. A.; Benkli, K.; Chevallet, P.; Akalin, G. Eur. J. Med. Chem. 2008, 43, 981.

10. Bekhit, A. A.; Ashour, H. M. A.; Abdel Ghany, Y. S.; Bekhit, A. E. A.; Baraka, A. Eur. J. Med. Chem. 2008, 43, 456.

11. Karegoudar, P.; Karthikeyan, M. S.; Prasad, D. J.; Mahal- inga, M.; Holla, B. S.; Kumari, N. S. Eur. J. Med. Chem. 2008, 43, 261.

12. Verma, A.; Saraf, S. K. Eur. J. Med. Chem. 2008, 43, 897.

13. Kumar, A.; Rajput, C. S. Eur. J. Med. Chem. 2009, 44, 83.

14. Misturu, O.; Atsushi, K.; Hiroyoshi, S.; Hisashi, T. Chem. Pharma Bull. 1995, 43, 947.

15. Kim, J. Y.; Seo, H. J.; Lee, S.-H.; Jung, M. E.; Ahn, K. W.; Kim, J. M.; Lee, J. H. Bioorg. Med. Chem. Lett. 2009, 19, 142.

16. Sidique, S.; Ardecky, R.; Su, Y.; Narisawa, S.; Brown, B.; Millan, J. L.; Sergienko, E.; Cosford, N. D. P. Bioorg. Med. Chem. Lett. 2009, 19, 222.

17. Collins, H. A.; Microbiological Methods, 2nd ed; Butterworth: London, 1976.

18. Methods for Dilution Antimicrobial Susceptibility Tests for Bacteria That Grow Aerobically NCCLS Approval Standards, Wayne, PA, USA, 2003.

19. NCCLS Approval Standard Document M2-A7, National Committee for Clinical Laboratory Standards, Vilanova, PA, USA, 2000. 